$\mathrm{e} 0640$

COMPARATIVE STUDY ON MANIPULATION AND IMAGING
OF 4FR VERSUS 6FR CATHETERS BY TRANSRADIAL CORONARY ANGIOGRAPHY

doi:10.1136/hrt.2010.208967.640

Fu Xianghua, Liu Jun, Wu Weili, Gu Xinshun, Li Shiqiang, Wang Xuechao. The 2nd Hospital of Hebei Medical University, Shijiazhuang, Hebei, China

Objective To prospectively compare the imaging quality of CAG and feasibility in manipulation with $4 \mathrm{Fr}$ versus $6 \mathrm{Fr}$ catheters by transradial CAG.

Methods A total of 866 consecutive patients who required coronary angioplasty were enrolled into this study. First, all patients underwent CAG with a 4Fr catheter by transradial approach, and 1 week later, underwent CAG with a 6Fr catheter before the coronary angioplasty by transradial approach. The handing, torque, selectivity, and stability with the 4Fr and 6Fr catheter were graded from 1 (excellent) to 4 (unacceptable) by the operator. The angiographic quality (QUAL) of CAG was also graded from 1 (unacceptable) to 10 (excellent) by two doctors in the catheter laboratory.

Results There were no statistical differences between the 4Fr catheter and 6Fr catheter in procedural time and fluoroscopy time, whereas compression time in the 4Fr catheter was significantly shorter $(p<0.01)$, bleeding volume was lower $(p<0.01)$, and the rate of accesssite complication was significantly lower $(p<0.01)$ compared with the $6 \mathrm{Fr}$ catheter. The feasibility scores of the left and right coronary catheter were similar in both catheter sizes ( $p>0.05)$. However, the feasibility scores of the pigtail catheter was significantly difference between the 4Fr and 6Fr catheter $\mathrm{p}<0.05$ ). QUAL using 4Fr or 6Fr catheters were equivalent $\mathrm{p}>0.05$ ). The total contrast volume was significantly less in the 4Fr catheter group $(p<0.05)$.

Conculsion CAG with 4Fr catheters is technically feasible; it reduces access-site complications after the procedure and the angiographic results were acceptable.

\section{E0641 CONTRAST STUDY OF TRANSULNAR ARTERY PCI AND TRANSRADIAL ARTERY PCI}

doi:10.1136/hrt.2010.208967.641

Fu Xianghua, Gu Xinshun, Fan Weize, Jiang Yunfa, Wei Yongyun, Liu Jun, Hao Guozhen, Li Shiqiang. The 2nd Hospital of Hebei Medical University, Shijiazhuang, Hebei, China

Objective To compare the feasibility and safety of TUA-PCI to TRAPCI (transradial artery PCI).

Methods A total of 320 patients from 38 to 80 years old were randomised into transradial artery PCI group and transulnar artery group. The time of manipulative duration for each procedure of PCI was recorded. The time of Allen's test, lumen diameter ( $\mathrm{mm})$, cross area of vessel lumen $\left(\mathrm{mm}^{2}\right)$, blood velocity (Vs max), blood flow resistance index in ulnar and radial artery were measured and recorded, respectively, as well were compared quantitatively before and after PCI procedure.

Result In each group the success rate of puncture of access artery was $98.7 \%$, because each group had two cases failed and transferred to another group. In PCI-TRU group 212 lesion segments of 178 vessels in all patients were angioplasticized successfully via TRU by $6 \mathrm{~F}$ guiding catheter and 215 stents were implanted. The average time of manipulative duration of guiding catheters engaging in ostium of target coronary artery, the average time of under x-ray fluoroscopy and the total time of the whole procedure were no significant difference between the PCI-TRU group and the PCI-TRA group. In PCI-TRA group 210 lesion segments of 177 vessels in were angioplasticized successfully via TRA by $6 \mathrm{~F}$ guiding catheter and 214 stents were implanted. When the introducer was taken off, with maintaining heparin infusion immediately after procedure, the access site in ulnar or radial artery was compressed by a tourniquet for $6 \mathrm{~h}$ and the compress pressure were gradually decreased, and it was unnecessary to limit the ambulance of the patients. The average hospital stay was no statistic significance between PCI-TRU group and PCI-TRA group ( $p>0.05$ ). No significant change was found in the parameters of the vessel diameter, blood velocity, cross area of vessel lumen, blood flow resistance index and the level of blood oxygen in finger and no complication such as occlusion of ulnar or radial artery in all patients following up 1 month after PCI procedure.

Conclusion Transulnar artery PCI was as safety and efficacy as transradial artery, the ulnar artery might be selected as one approach of antebrachial artery for PCI in patients with coronary heart disease.

\section{e0642 EFFECT OF INTRACORONARY ANISODAMINE ON NO- REFLOW PHENOMENON AFTER PCI IN MINI-PIG WITH AM}

doi:10.1136/hrt.2010.208967.642

Fu Xainghua, Gu Xinshun, Liu Jun, Fan Weize, Jiang Yunfa, Wang Yanbo, Hao Guozhen, Li Shiqiang. The 2nd Hospital of Hebei Medical University, Shijiazhuang, Hebei, China

Objective To evaluate the effect of intracoronary anisodamine to correct the no-reflow phenomenon during AMI on no-reflow minipig model made by superselective $\mathrm{LAD}$ with $4 \mathrm{~F}$ catheter then injection of micro thrombus.

Method 14 no-reflow minipig models were randomised to intracoronary saline $(4 \mathrm{ml})$, diltiazem (1 $\mathrm{mg}$ diluted to $4 \mathrm{ml}$ ) and anisodamine (2 $\mathrm{mg}$ to $4 \mathrm{ml}$ ) groups. CAG was performed at 1, 3, 5, 10th minute after intracoronary injection. Corrected TIMI frame count and coronary artery diameter were obtained by OCA, meanwhile ECG and homodynamic parameters (HR, LVEDP, intracoronary MAP,) were recorded.

Results The corrected TIMI frame count in Anisodamine group was significantly decreased by $13.2 \%, 25.3 \%, 35.6 \%, 33.6 \%$ at $1,3,5,10$ th minute compared with saline group, while decreased by $10.6 \%$, $20.4 \%, 14.3 \%, 21.5 \%$ at $1,3,5,10$ th minute compared with diltiazem group, (respectively). The medial LAD diameter in Anisodamine group increased from $2.12 \pm 0.38 \mathrm{~mm}$ to $2.60 \pm 0.43 \mathrm{~mm} \quad(\mathrm{p}<0.05)$ while to $2.51 \pm 0.42 \mathrm{~mm}$ in diltiazem group as compared before $(p<0.05)$. HR and intracoronary MAP were increased and LVEDP reduced at $1,3,5$, 10th minute after administration of anisodamine, which had significance compared with group NS (125 \pm 20 vs $140 \pm 25 \mathrm{bpm}, 95 \pm 12$ vs $126 \pm 13 \mathrm{~mm} \mathrm{Hg}, \mathrm{p}<0.05)$, accompanied with HR increasing $(p<0.05)$. It was very important that no serious side effect and toxic reaction were found.

Conclusions Intracoronary anisodamine might reduce and correct the no-reflow phenomenon after PCI-AMI with the dilation of coronary vessel and homodynamic improvement, as well as no serious side effect and toxic reaction.

\section{e0643 EFFECTS OF NO-REFLOW PHENOMENON IDENTIFIED BY MYOCARDIAL BLUSH GRADES ON SYSTOLIC FUNCTION AND SYNCHRONY IN PATIENTS WITH ACUTE MYOCARDIAL INFARCTION AFTER PCI}

doi:10.1136/hrt.2010.208967.643

Fu Xianghua, Liu Jun, Wu Weili, Gu Xinshun, Li Shiqiang, Wang Yanbo. The 2nd Hospital of Hebei Medical University, Shijiazhuang, Hebei, China

Objective To investigate the effect of no-reflow phenomenon after percutaneous coronary intervention (PCI) in patients with acute myocardial infarction (AMI).

Methods A total of 128 patients with the first AMI of the anterior wall were involved in this study. All patients underwent coronary 